\title{
Polystyrene/calcium phosphate nanocomposites: Contact angle studies based on water and methylene iodide
}

\author{
S. P. Thomas ${ }^{1,2}$, S. Thomas ${ }^{1 *}$, R Abraham ${ }^{3}$, S. Bandyopadhyay 4 \\ ${ }^{1}$ School of Chemical Sciences, Mahatma Gandhi University, P. D. Hills P.O., Kottayam-686560, Kerala, India \\ ${ }^{2}$ Visiting Research Associate, School of Materials Science and Engineering, University of New South Wales, \\ Sydney-2052, Australia \\ ${ }^{3}$ Department of Physics, St. Dominic's College, Kanjirappilly, Kottayam, Kerala, India \\ ${ }^{4}$ School of Materials Science and Engineering, University of New South Wales, Sydney-2052, Australia
}

Received 27 April 2008; accepted in revised form 15 June 2008

\begin{abstract}
Polystyrene (PS) based nanocomposites were prepared by melt mixing in a Brabender Plasticoder with calcium phosphate as the nanofiller. These nanocomposites showed improvement in almost all physical properties. Contact angle measurements of the composites with water and methylene iodide were carried out. Various contact angle parameters such as total solid surface free energy, work of adhesion, interfacial free energy and spreading coefficient were analysed. The interaction parameter between the polymer and the liquids has been calculated using the Girifalco-Good's equation.
\end{abstract}

Keywords: nanocomposites, polystyrene, calcium phosphate, contact angle measurements

\section{Introduction}

Nanocomposites are a new class of polymer materials with an ultrafine phase dispersion of the order of a few nanometers that shows very interesting properties often very different from those of conventional filled polymers [1-4]. The presence of these nanoparticles improves the elastic modulus without decreasing the elongation at break and does not worsen the rheological and processing behavior and the optical properties of the polymer matrix. The main problem in the preparation of nanocomposites in the molten state [5-7] is the good filler dispersion the polymer matrix. All properties have been measured considering a possible use of these new materials for several different applications.

Contact angle measurements are often used as an empirical indicator of 'wettability' and interfacial tension. True contact angle determination of a liquid-solid-vapor system satisfies Young's equation, where the wetting tension $\left(\gamma_{l v} \cos \theta\right)$ is equivalent to the solid surface tension $\left(\gamma_{s v}\right)$ minus the liquidsolid interfacial tension $\left(\gamma_{l v}\right)$ [8]. However, in most cases the contact angle exhibits hysteresis for advancing and receding liquids is due to the roughness and heterogeneity of a solid surface [9-11]. Therefore, in practical usage, the measured angle is always considered an 'apparent' contact angle. The most popular method for direct measurement of the static contact angle is the sessile drop method, where the angle is determined from the tangent made to the drop curvature at the base.

For polymer production where particulates or fibers are used for reinforcement, colorant, flame retardancy or stability, understanding the wetting phenomena has considerable value in relation to the material performance. Lowering the free energy of the system, the polymer chains must preferentially interact with the filler surface, where wettability

*Corresponding author, e-mail: sabut@sancharnet.in and sabut552001@yahoo.com

(C) BME-PT and GTE 
plays a dominant role in successfully achieving the desired structure $[12,13]$. Park and Jin studied the effects of silane coupling agent treatments on the fiber surface properties in terms of the surface energetics of the fibers and the mechanical interfacial properties, including Interlaminar Shear Strength (ILSS) and fracture toughness $\left(K_{\mathrm{Ic}}\right)$, of the unsaturated polyester composites [14]. Saihi et al. performed the wettability studies on polyethylene terephthalate (PET) fibres according to the Wilhelmy method [15]. They obtained qualitative and quantitative indications about the degree of the water and oil repellency and of the surface free energy of the grafted surface. Yeh et al studied the contact angle behaviour of the Acrylonitrile butadiene styrene (ABS) clay nanocomposites very recently [16]. Contact angle of water on the ABS-clay nanocomposite materials increased with respect to the increase in clay content.

Several other reports on the wetting phenomena on the polymer nanocomposites appeared in the literature recently [17-21]. Here our aim was to study the wetting behaviour of the polystyrene/calcium phosphate nanocomposites with respect to water and methylene iodide. This study focuses on the effect of weight percentage of nanofiller on wetting characteristics such as work of adhesion, total surface free energy, interfacial free energy and spreading coefficient.

\section{Experimental}

\subsection{Materials}

Atactic polystyrene was obtained from Polychem Bombay, India. The specific properties of the polymer are denoted in Table 1.

\subsection{Nanoparticle preparation}

The nanosized calcium phosphate filler particles were synthesized using in situ deposition technique

Table 1. Characteristics of polystyrene

\begin{tabular}{|l|c|}
\hline \multicolumn{1}{|c|}{ Property } & Value \\
\hline Density [g/cc] & $1.04-1.065$ \\
\hline Poisson's ratio & 0.33 \\
\hline Solubility parameter $\left[\left(\mathrm{cal} / \mathrm{cm}^{3}\right)^{1 / 2}\right]$ & 18.6 \\
\hline Water absorption $(\mathrm{ASTM})$ & $0.05 \%$ \\
\hline Softening temperature $\left[{ }^{\circ} \mathrm{C}\right]$ & $108^{\circ} \mathrm{C}$ \\
\hline Molecular weight $\left(M_{w}\right)$ & 218000 \\
\hline
\end{tabular}

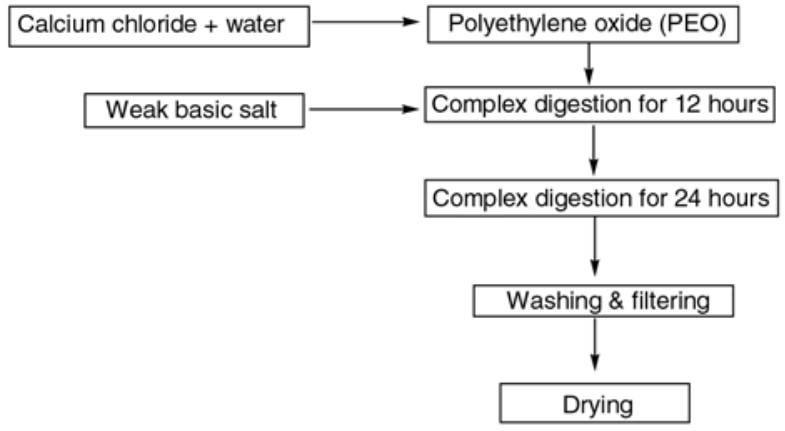

Figure 1. Schematic representation of the nanoparticle synthesis

in the presence of polyethylene oxide (PEO) as follows: Firstly, a complex of calcium chloride $\left(\mathrm{CaCl}_{2}\right)$ with $\mathrm{PEO}$ was prepared in desired proportions in methanol. An appropriate stoichiometric amount of trisodium phosphate in distilled water was added to the above complex slowly without stirring. The whole mixture was allowed to digest at room temperature for $24 \mathrm{~h}$ when both the chloride and phosphate ions diffused through the PEO and formed a white gel like precipitate, which was filtered, washed and dried. The concentrations of $\mathrm{PEO}-\mathrm{CaCl}_{2}$ complex were varied in the range from $2: 1,4: 1,8: 1,16: 1$ and 32:1 (molar ratio) respectively. The yield of the calcium phosphate was recorded as 83 and $62 \%$ for $4: 1$ and $16: 1$ ratios respectively. The method of nanoparticle synthesis is given in Figure 1. The particle size was measured by X-ray diffractogram and transmission electron microscopy techniques. X-ray diffractograms of the particles were measured using Philips $1140 \mathrm{X}$-ray irradiation apparatus using $\mathrm{CuK}_{\alpha}$ radiation with $\lambda=1.5406 \AA$ at room temperature. TEM images of the particles and composites were measured by Philips CM200 field emission gun TEM using thin transparent sections. Nanoparticles with $\sim 10 \mathrm{~nm}$ size was used for further studies (these were prepared by taking $\mathrm{PEO}$ and $\mathrm{CaCl}_{2}$ in the ratio 2:1).

\subsection{Nanocomposite preparation}

The nanoparticles were incorporated into polystyrene by melt blending technique using a Brabender Plasticoder at $200^{\circ} \mathrm{C}$ with varying filler amount as $1,3,5,7$ and $10 \mathrm{wt} \%$. The various composites are designated as P0, P1, P3, P5, P7 and P10 respectively. 


\subsection{Contact angle measurements}

Contact angle measurements were carried out in a FTA 100 Series (First Ten Angstroms, Portsmouth, Virginia 23704 USA). Measurements were carried out with water (triply distilled) and methylene iodide (Kemphasol, Bombay) on samples of size $1 \times 1 \times 2 \mathrm{~cm}^{3}$ at room temperature. The volume of the sessile drop was maintained as $5 \mu \mathrm{l}$ in all cases using a microsyringe. The contact angle was measured within 45-60 s of the addition of the liquid drop with an accuracy of $\pm 1^{\circ}$. Measurements were repeated six to ten times with different test pieces of the same sample to check the accuracy. Also contact angles were measured with definite time intervals for a single drop and the measurements were recorded as snap shots.

\subsection{Theory and calculations}

It is well known that the knowledge of surface energetics at a given temperature of a solid has recently allowed significant progress in many academic and scientific fields involving two, not identical, molecular interactions at a certain intermolecular distance, such as adsorption (gas-solid), wettability (liquid-liquid), and adhesion (solid-solid) [22, 23]. The basic equation relating the surface free energy of a solid $\left(\gamma_{s}\right)$ and that of the liquid in contact with the solid $\left(\gamma_{l}\right)$, the interfacial free energy between the solid and the liquid $\left(\gamma_{s l}\right)$, and the contact angle $(\theta)$ is due to Young [24]. It is given by Equation (1):

$\gamma_{l} \cos \theta=\gamma_{s}-\gamma_{s l}$

In Equation (1), $\gamma_{s}$ and $\gamma_{s l}$ are not amenable to direct measurement. Plot of $\cos \theta$ against the surface tension for a homologous series of liquids, $\gamma_{l}$, can be extrapolated to give a critical surface tension, $\gamma_{c}$, at which $\cos \theta=1$ [25]. $\gamma_{c}$ has been taken as an approximate measure of the surface energy, $\gamma_{s}$, of the solid. However, a limitation of this consideration is that the precise value of $\gamma_{c}$ depends on the particular series of liquids used to determine it.

A more appropriate method has been presented by Fowkes considering solid dispersion forces using a geometric mean equation [26]. Later, Owens and Wendt [27] and Kaelble [28] modified Fowkes equation further by also assuming the polar attraction forces which also included the hydrogen bonding forces. $\mathrm{Wu}[29]$ has found a still better agree- ment to obtain $\gamma_{s}$ when he used a harmonic mean equation which combines both the dispersion and the polar forces. Wu's approach has been quite satisfactoriy verified by several authors [30-34]. In order to verify Wu's approach, two liquids of dissimilar polarity are selected. Water and methylene iodide have been reported to be a useful pair of liquids for obtaining $\gamma_{s}$ of polymers [35]. Wu's harmonic mean equations for water and methylene iodide are written by Equation (2):

$$
\left(1+\cos \theta_{w}\right) \gamma_{w}=4\left[\frac{\gamma_{w}^{d} \gamma_{s}^{d}}{\gamma_{w}^{d}+\gamma_{s}^{d}}+\frac{\gamma_{w}^{p} \gamma_{s}^{p}}{\gamma_{w}^{p}+\gamma_{s}^{p}}\right]
$$

and by Equation (3):

$$
\left(1+\cos \theta_{m}\right) \gamma_{m}=4\left[\frac{\gamma_{m}^{d} \gamma_{s}^{d}}{\gamma_{m}^{d}+\gamma_{s}^{d}}+\frac{\gamma_{m}^{p} \gamma_{s}^{p}}{\gamma_{m}^{p}+\gamma_{s}^{p}}\right]
$$

where the subscripts $d$ and $p$ stand for contributions due to dispersion and polar forces respectively. Data for water and methylene iodide were taken from the literature [27].

Water: $\gamma_{w}=72.8 \mathrm{~mJ} / \mathrm{m}^{2}, \gamma_{w}^{d}=21.8 \mathrm{~mJ} / \mathrm{m}^{2}$,

$$
\gamma_{w}^{d}=51.0 \mathrm{~mJ} / \mathrm{m}^{2}
$$

Methylene iodide: $\gamma_{m}=50.8 \mathrm{~mJ} / \mathrm{m}^{2}$,

$$
\begin{aligned}
& \gamma_{m}^{d}=49.5 \mathrm{~mJ} / \mathrm{m}^{2}, \\
& \gamma_{m}^{p}=1.3 \mathrm{~mJ} / \mathrm{m}^{2}
\end{aligned}
$$

$\gamma_{s}^{d}$ and $\gamma_{s}^{p}$ for different compositions of nanocomposites were determined by solving Equations (2) and (3) with the help of a $\mathrm{C}$ programme.

The total solid surface free energy is represented by Equation (4):

$\gamma_{s}=\gamma_{s}^{d}+\gamma_{s}^{p}$

The work of adhesion, $W_{A}$, was calculated using Equation (5):

$W_{A}=(1+\cos \theta) \gamma_{l}$

where $\gamma_{l}$ is the surface tension of the liquid used for the contact angle measurement.

The interfacial free energy, $\gamma_{s l}$, was calculated from Dupré's Equation (6) [35]:

$\gamma_{s l}=\gamma_{s}+\gamma_{l}-W_{A}$

The spreading coefficient, $S_{c}$, was determined from Equation (7) [35]:

$S_{c}=\gamma_{s}-\gamma_{s l}-\gamma_{l}$ 
Girifalco-Good's interaction parameter, $\phi$, between the polymer and the liquid was determined using Equation (8) [36]:

$$
\phi=\frac{\gamma_{l}(1+\cos \theta)}{2\left(\gamma_{l} \gamma_{s}\right)^{\frac{1}{2}}}
$$

\section{Results and discussion}

\subsection{Nanoparticle characterization}

Nanoparticles of calcium phosphate were characterized by XRD as well as TEM techniques. Figure 2(a-f) corresponds to the XRD patterns of nanoparticles obtained, (a) corresponds to the calcium phosphate without $\mathrm{PEO}$ and $(\mathrm{b}-\mathrm{f})$ for calcium phosphate with PEO concentration from 2:1 to 32:1 respectively. In the figure, there are a number of crystalline forms reported for calcium phosphate existing in both anhydrous and hydrated states. The calcium phosphate without any polymer shows

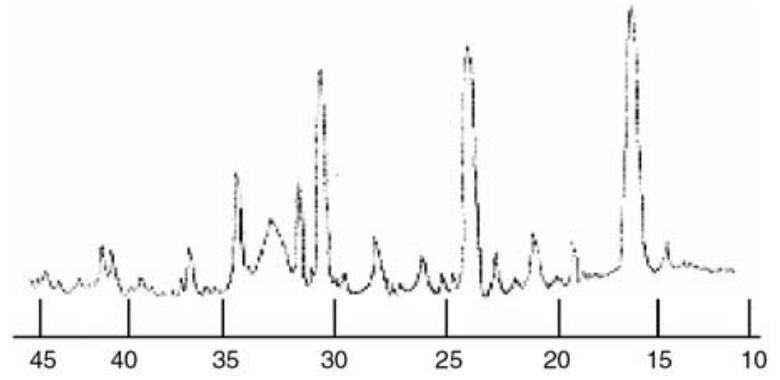

a)

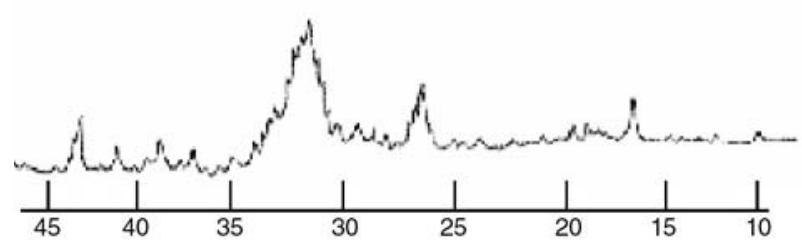

c)

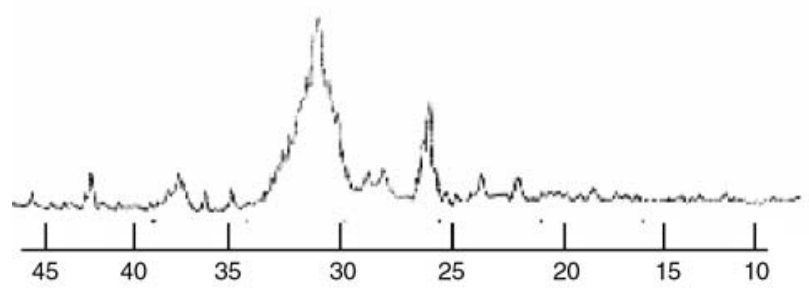

e)
3 types of phases. Most of the peaks in diffraction patterns correspond to $\beta$ calcium orthophosphate (major phase) while the minor phase contains calcium orthophosphate primary along with small amount of calcium metaphosphate hydrate respectively. With increase of PEO concentration, there occurs a suppression of peaks in the diffraction patterns compared to curve a. A large number of peaks are suppressed and peaks at $2 \theta$ of $31.9^{\circ}$ broaden with increase of PEO concentrations from 42 to $89 \%$. Only two peaks are clearly seen. The peak at $31.9^{\circ}$ corresponds to all the three states $\left(\mathrm{Ca}_{3}\left(\mathrm{PO}_{4}\right)_{2}\right.$, $\left.\mathrm{Ca}_{3}\left(\mathrm{H}_{2} \mathrm{PO}_{4}\right)_{2}, \mathrm{Ca}_{3}\left(\mathrm{PO}_{3}\right)_{2} \cdot 3 \mathrm{H}_{2} \mathrm{O}\right)$ and at $25.1^{\circ}$ corresponds to the minor phase $\mathrm{Ca}_{3}\left(\mathrm{H}_{2} \mathrm{PO}_{4}\right)_{2}$. It is interesting to note the extent of broadening, from 42 to $89 \%$. This shows the small size of crystals, which could be associated with a good molecular level mixing. Thus on comparing curve a with curve $b$ to $f$ in Figure 2, it is obvious that the effect of polymer plays a prominent role in the structure and growth behaviour of calcium phosphate crystals (in the curves the $y$ axis indicates the intensity). Since

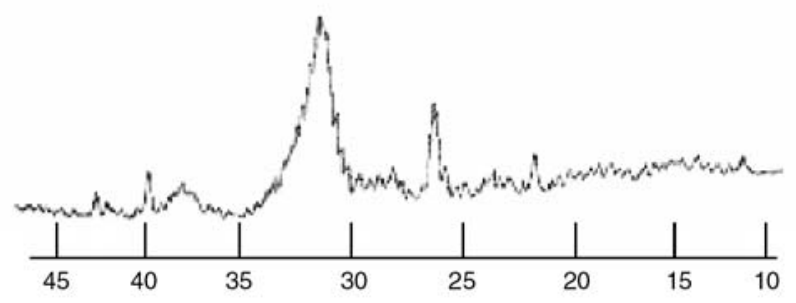

b)

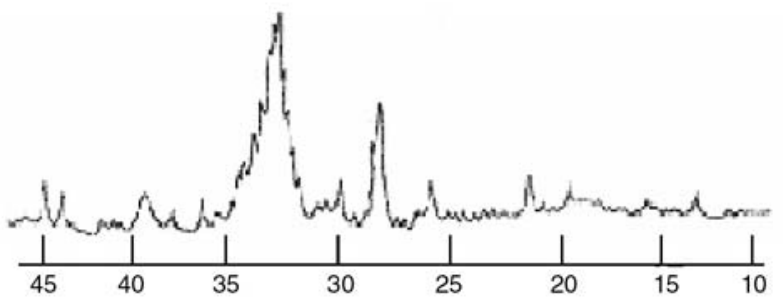

d)

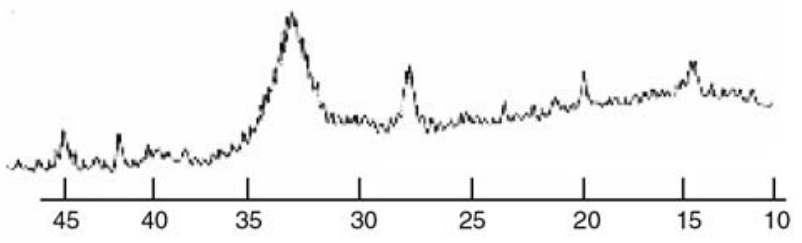

f)

Figure 2. XRD patterns for the calcium phosphate nanoparticles (a) Calcium Phosphate without PEO. The PEO: $\mathrm{CaCl}_{2}$ ratio in (b) 2:1, (c) 4:1, (d) 8:1 (e) 16:1 and (f) 32:1 (in the $x$ axis $2 \theta$ and in $y$ axis intensity is plotted) 
$\mathrm{CaCl}_{2}$ is first complexed with $\mathrm{PEO}$, only certain crystalline phases of calcium phosphate are allowed to grow as compared to a large number of phases getting developed in normal free precipitation. From the XRD patterns, the crystallite size was calculated using Scherrer formula, see Equation (9):

$$
d[\AA]=\frac{k \lambda}{\Delta 2 \theta \cdot \cos \theta}
$$

where $k$ is the order of reflection, $\lambda$ is $1.542 \AA$, the diffraction angle and $\Delta 2 \theta$ is the full width at half maximum (FWHM). As the concentration of the PEO increases the particle size decreases. The corresponding data obtained from XRD measurements is given in Table 2.

The TEM images of the particles are shown in Figures $3(\mathrm{a}-\mathrm{c})$, which are taken for ratios of 2:1, 4:1 and 32:1 respectively. The particles were made a suspension in alcoholic medium and the images were taken after laying the particles on a copper grid. The alcohol evaporated and the particles could be seen clearly in the images. The particle size distributions for each concentration were calculated

Table 2. XRD values for the calcium phosphate nanoparticles

\begin{tabular}{|c|c|c|}
\hline (PEO: $\mathrm{CaCl}_{2}$ ) & $\begin{array}{c}\text { FWHM }(2 \theta) \\
\text { radians }\end{array}$ & $\begin{array}{c}\text { Particle size (L) } \\
\text { nm }\end{array}$ \\
\hline Calcium phosphate (a) & 0.00174 & 82.4 \\
\hline $2: 1(b)$ & 0.0134 & 10.65 \\
\hline $4: 1(\mathrm{c})$ & 0.0156 & 9.24 \\
\hline $8: 1(d)$ & 0.0162 & 8.96 \\
\hline 16:1 (e) & 0.0176 & 8.02 \\
\hline 32:1 (f) & 0.0181 & 7.82 \\
\hline
\end{tabular}

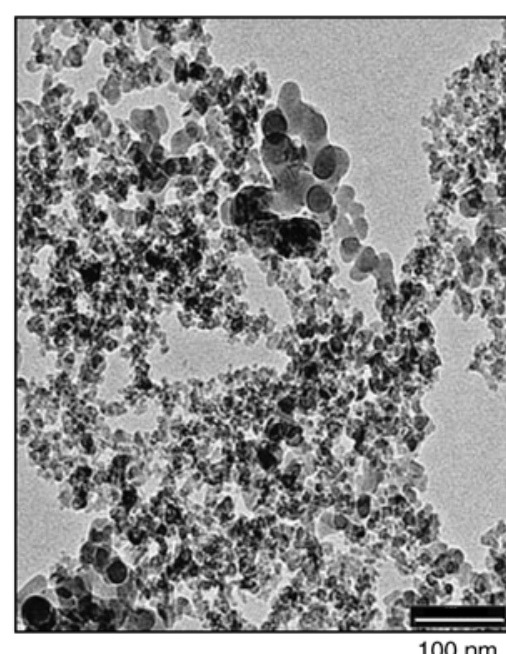

a)

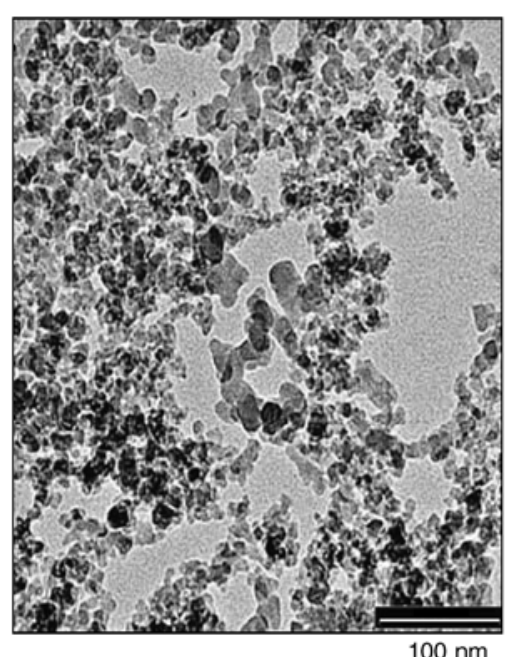

b) using image analyzer software. On an average 400 particles were measured at a time to calculate the particle size distribution. The distribution was found to be narrow. The particle size reduction against concentration of PEO is shown in Figure 4. The particle size reduction is attributed to the increase in the amount of oxygen atoms available from PEO matrix as we increase the concentration [37]. The reaction between calcium and phosphate ions is taking place in the 'oxygen cage' available from the PEO molecules. On increasing the concentration of the PEO molecules the number of oxygen atoms increases which will increase the amount of 'oxygen cage' considerably. This enables the suppression of the formed particles and reduces the size further. The yield of the calcium phosphate was recorded as 83 and $72 \%$ for $4: 1$ and 16:1 ratios respectively. Calcium phosphate nanoparticles obtained with the concentration ratio 4:1

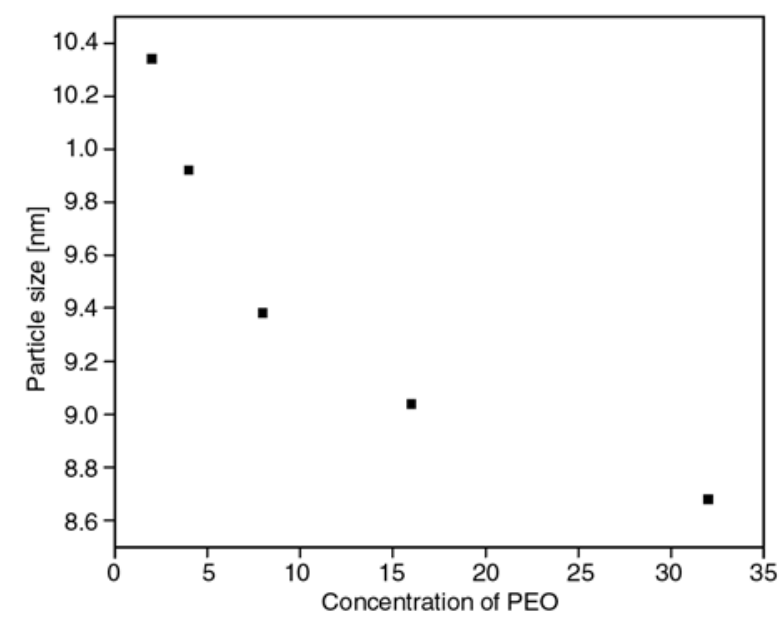

Figure 4. Effect of PEO concentration on particle size of calcium phosphate

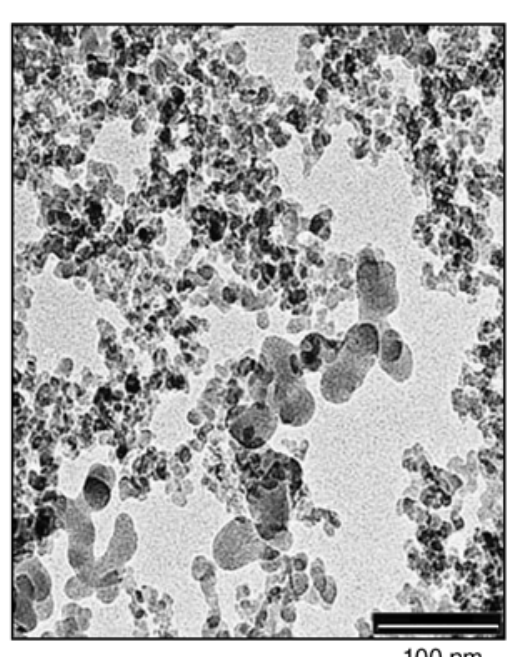

c)

Figure 3. TEM image of the calcium phosphate nanoparticles (PEO: $\mathrm{CaCl}_{2}$, (a) 2:1, (b) 4:1, (c) 32:1) 
(particle size approximately $10 \mathrm{~nm}$ ) were exclusively used for this study.

\subsection{Characterization of composites}

TEM images of the thin sections of the composites were analysed to see how the particles are dispersed in the matrix for the composites with 3, 5, 7 and $10 \%$ respectively (Figure 5(a-d)). TEM will be required to tell if nano-dispersion has been achieved. Generally, the low magnification images indicate how well-dispersed the filler is, while the high magnification images permits the identification of an intercalated or delaminated morphology in polymer nanocomposites. It is quite clear that filler is well distributed in all the styrenic polymers from the TEM images. Discrete particles of nano fillers were seen in the initial compositions (Figure 5(a and $b)$ ). Good dispersion of fillers in to the matrix can be seen for the lower concentrations. However, it can be seen from the images, as the concentration of the filler increased, the efficient incorporation is lacking mainly due to the filler agglomeration. We can see that the particles get agglomerated upon increasing the amount of filler.

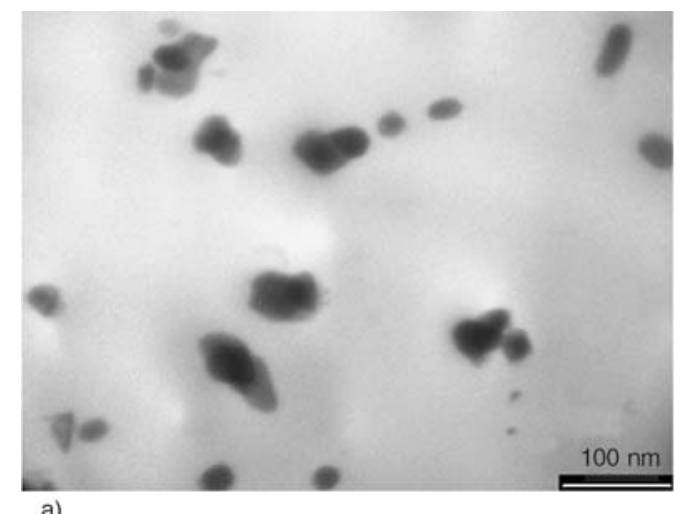

a)

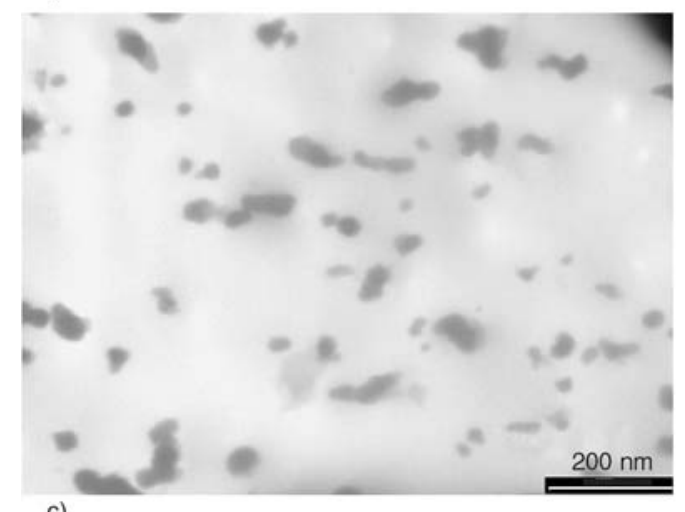

c)

\subsection{Contact angle measurements}

Contact angle measurements of nanocomposites of polystyrene with calcium phosphate were done at room temperature with water and methylene iodide as the liquids. Various parameters from these measurements were calculated. At first, the contact angles were measured for each specimen for at least six to ten times. The average is taken as the contact angle for the particular specimen. Figure 6 shows the representative pictures of the measurements with methylene iodide as the liquid. In 6(a) the contact angle for the neat polystyrene is given and 6(b), (c) and (d) represents the same for P3, P5 and P10 respectively. Here the tilt angle is always kept as 0 and an average of the left hand side and right hand side contact angles is given as the true contact angle. In most of the measurements the left and right hand side values are similar. The measurements were done at room temperature with respect to the filler loading. For water the neat polymer show a contact angle equal to $90^{\circ}$. While increasing the filler content the contact angles decreased to around $78^{\circ}$ as shown in Figure 7. Polystyrene is basically a hydrophobic polymer and thus the high value of contact angle is justifiable. Calcium phos-

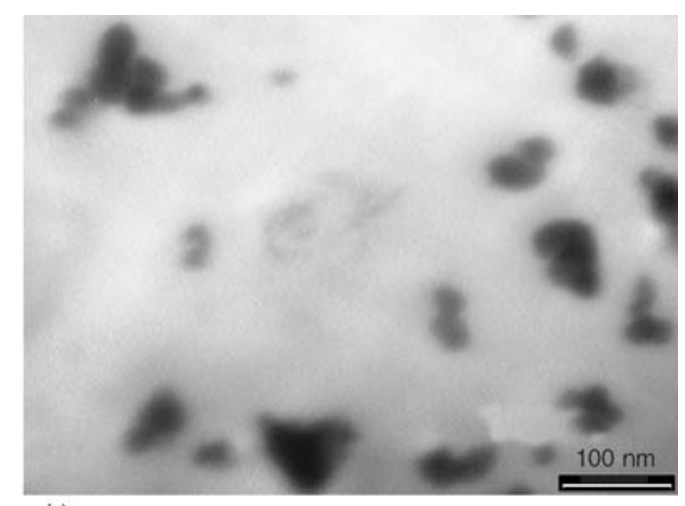

b)

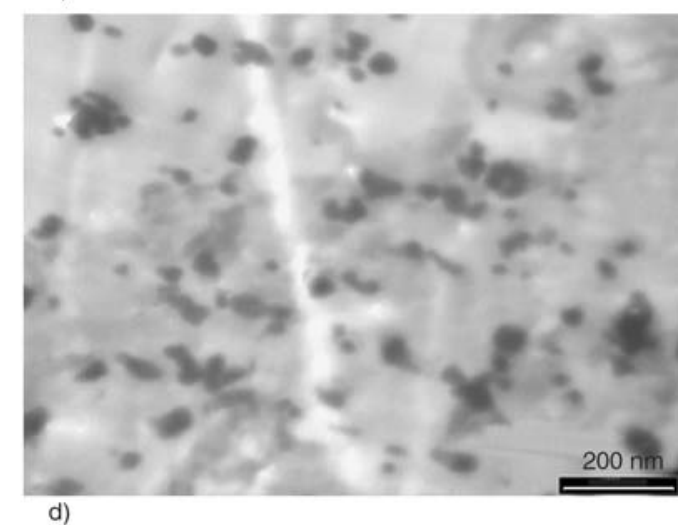

Figure 5. TEM images of polystyrene nanocomposites: a) P3, b) P5, c) P7 and d) P10 (a and b magnification is $100 \mathrm{~nm}$, c and $\mathrm{d}$ magnification is $200 \mathrm{~nm}$ ) 


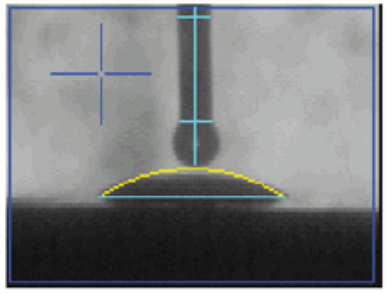

a) (32.93)

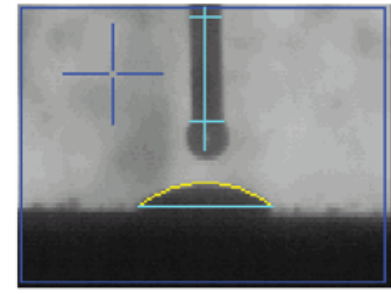

b) (38.69)

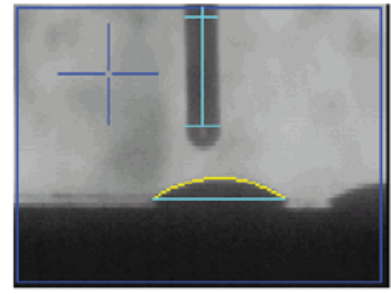

c) (36.05)

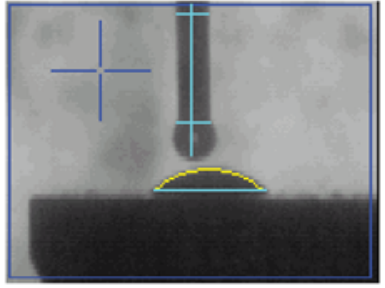

d) (43.69)

Figure 6. Representative figures of contact angle measurements of (a) P0 (b) P3 (c) P5 and (d) P10 with methylene iodide as liquid. Corresponding contact angles are given in parenthesis

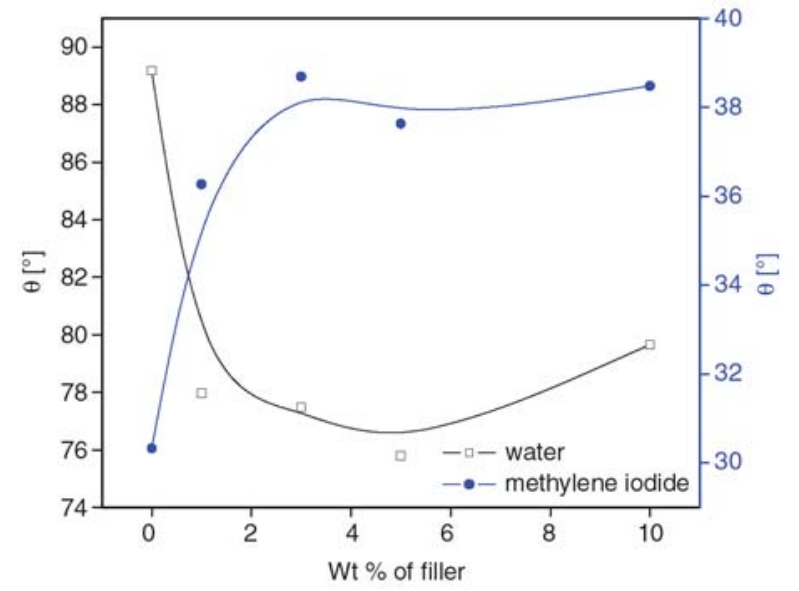

Figure 7. Variation of contact angle of water and methylene iodide with respect to filler loading

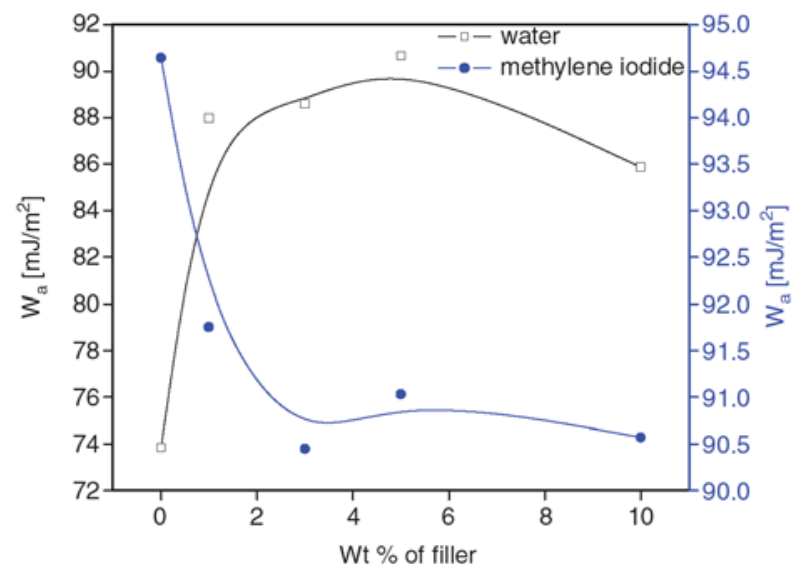

Figure 8. Variation of work of adhesion of water and methylene iodide with respect to filler loading

phate incorporation into the matrix decreases the contact angle. This means that the hydrophilicity is increased for polystyrene. The surface of the composites contains some of the nanofillers added and this eventually decreased the hydrophobic nature. But the amount of fillers has not much effect on the contact angle. For all the composites the contact angles remains almost constant around $78^{\circ}$. In the case of methylene iodide also the hydrophilic nature of the matrix increases. For the neat polymer surface the contact angle is around $30^{\circ}$. On the addition of nanofillers it increased to around $38^{\circ}$ for all compositions. Both these results complement each other.

The work of adhesion, $W_{A}$, for the nanocomposites for both liquids is shown in Figure 8. $W_{A}$ is actually the work required to separate the solid and liquid depends solely on the contact angle and the surface tension of the liquid. Thus the $W_{A}$ for water increases and for methylene iodide shows the reverse trend. The magnitude of difference between the neat polymer and the nanocomposites are much higher for water compared to methylene iodide. Generally the work of adhesion can be correlated to the filler matrix interaction of filler with a liquid comparable with the matrix polymer. Here we try to correlate the work of adhesion of the nanocomposites with the filler matrix interaction even though the liquids selected do not mimic the virgin polymer completely. The TEM figures showed the efficient dispersion of nano fillers up to $5 \%$ and agglomeration for $10 \%$ loading (Figure $5(\mathrm{a}-\mathrm{d})$ ). Thus the effective dispersion of nanofillers into the matrix might have caused increase in the work of adhesion which is the work required to separate the liquid from the solid surface.

Figure 9 shows the variation in total solid surface free energy, $\gamma_{s}$, with respect to the filler loading. When we increase the filler loading the total solid surface free energy increases which means that the wetting of liquids is high compared to the neat specimens. Also the dispersive and polar components were calculated by solving the harmonic mean equations given earlier. For neat PS the $\gamma_{s}$ value was 46.3 and it increased up to 48.6 for the $5 \%$ filled composites. This means that the nature of the forces acting on the surface of the composites is different. From Table 3, we can see that the $\gamma_{s}^{d}$ values decreased compared to the neat polymer for all 
Table 3. Surface free energy and Girifalco-Good's interaction parameter of the nanocomposites

\begin{tabular}{|c|c|c|c|c|c|}
\hline Composites & $\gamma_{s}^{d}$ & $\gamma_{s}^{p}$ & $\gamma_{s}$ & $\phi_{w}$ & $\phi_{m}$ \\
\hline P0 & 41.7 & 4.5 & 46.3 & 0.63 & 0.97 \\
\hline P1 & 38.9 & 9.2 & 48.1 & 0.74 & 0.93 \\
\hline P3 & 37.9 & 9.6 & 47.5 & 0.75 & 0.92 \\
\hline P5 & 38.3 & 10.2 & 48.6 & 0.76 & 0.91 \\
\hline P10 & 38.0 & 8.6 & 46.7 & 0.73 & 0.93 \\
\hline
\end{tabular}

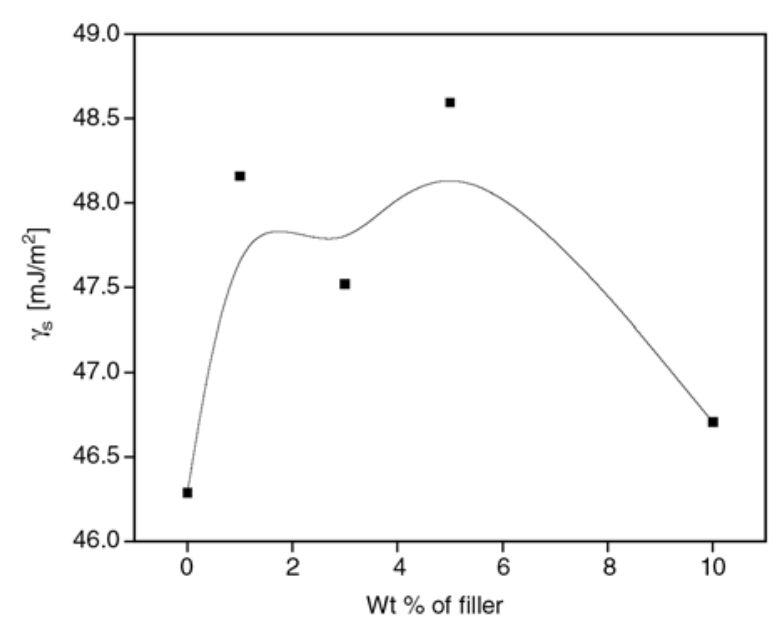

Figure 9. Variation of total solid surface free energy with respect to filler loading

compositions while $\gamma_{s}^{p}$ values show much difference from neat matrix. The polar forces acting on the surface of the composites increased compared to the neat polymer and thus the total solid surface free energy increased. Again for the $10 \%$ filled systems the $\gamma_{s}^{p}$ value is slightly lower compared to other filled compositions, which accounted for the decrease in the total solid surface free energy. The filler dispersion in the polymer matrix may also have affected the surface properties. Here the initial loadings have good dispersion behaviour while particle agglomeration occurred in the higher loading. This might have caused the decrement in $\gamma_{s}^{p}$ values for the $10 \%$ loading. So the hydrophilic nature of the composites became more prominent with the nanofiller addition. On the whole, the $\gamma_{s}$ values show not much difference for all the compositions. The interfacial free energy between the polymer surface and the test liquids, water and methylene iodide, were calculated and the curves are shown in Figure 10. The behaviour of the curves is just opposite to each other as one is polar and the other is non-polar. For water, $\gamma_{s w}$, decreases with respect to the filler loading and shows a minimum for 5\% filled system. For methylene iodide, $\gamma_{s m}$, increase

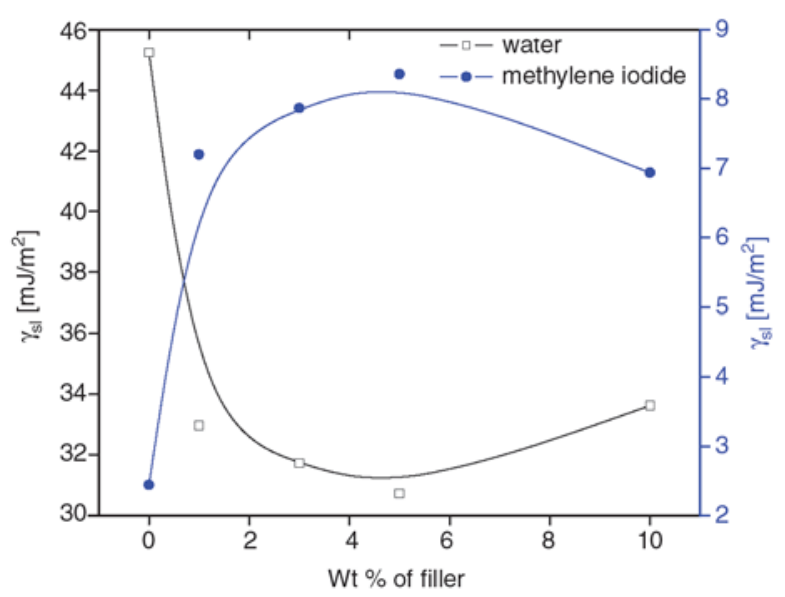

Figure 10. Variation of interfacial free energy of water and methylene iodide with respect to filler loading

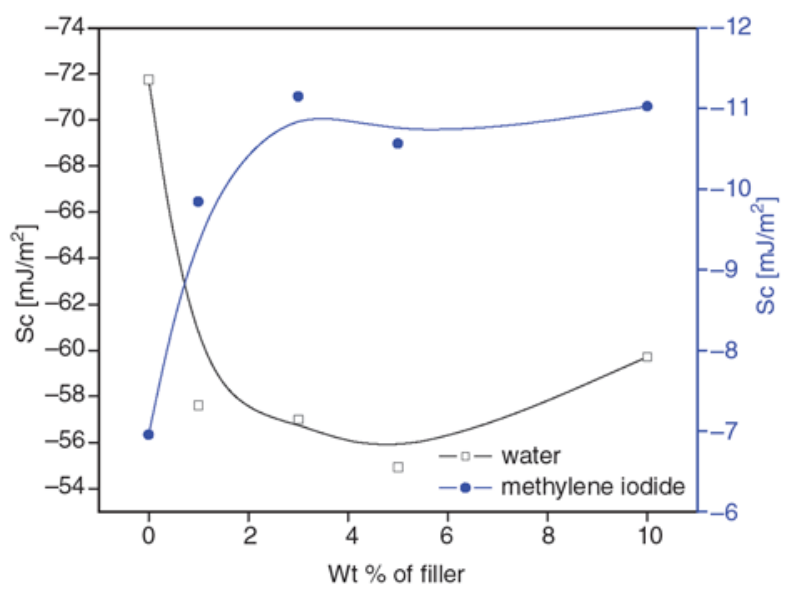

Figure 11. Variation of spreading coefficient of water and methylene iodide with respect to filler loading

from the neat sample, reaches a maximum for $5 \%$ filled systems and decrease for the higher filled systems.

In Figure 11 the spreading coefficient of the liquids; according to Equation (7); with respect to the filler loading is given. If the value is positive, the implication is that the liquid will spontaneously wet and spread on a solid surface and if it is negative the lack of wetting and spreading can be ascertained. This means the existence of a finite contact 
angle $(\theta>0)$. From Figure 11, we can deduce that as we increase the filler content the spreading coefficient values for water become less negative and for methylene iodide the reverse is true. Thus, the wetting increased for water with the addition of fillers indicating more hydrophilic nature. In the case of methylene iodide spreading coefficient became more negative and wetting will be difficult on the surface. Comparing water and methylene iodide, the less negative value is given by methylene iodide, which means that it is a better wetting agent for the current composites.

In order to understand the degree of interaction between the test liquid and polymer surface, Girifalco-Good's interaction parameter was calculated using the Equation (8) and the values are given in Table 3. Generally a higher value indicates greater interaction and vice versa. $\phi_{w}$ and $\phi_{m}$ are the Girifalco-Good's interaction parameters due to water and methylene iodide, respectively. From the values one can see that the interaction between methylene iodide and polymer surface is more compared to water and the surface. For water as we increase the filler loading the parameter show increase and for methylene iodide the interaction parameter show a slight decrease. Thus, for the polar liquid the interaction between the polymer surface and liquid increase while the opposite is shown for the non-polar liquid. This is can be evidenced also from Figure 10 showing the behaviour of interfacial free energy of the composites for both the liquids.

The variation of the contact angle of water with time on the surface of the neat PS and nanocomposites were analysed. The curves are shown in Figure 12. All curves show similar behaviour. In the initial region we can see a sharp decrease in contact angle. Thereafter the contact angles regularly decrease and after a long time it levels off. The surface of the specimens and the liquid have some interaction over a time of period and after reaching a saturation point the value becomes constant. The surface free energy of polymers and polymer composites decays due to the conformational alterations and surface restructuring as the contact time of the liquid increases [38, 39]. Lavielle and Schultz [38] have noted in acrylic grafted polyethylene samples undergo surface free energy changes when it is in long-time contact with water. In this case $\gamma_{s}^{d}$ initially increased and then decreased, $\gamma_{s}^{d}$ whereas

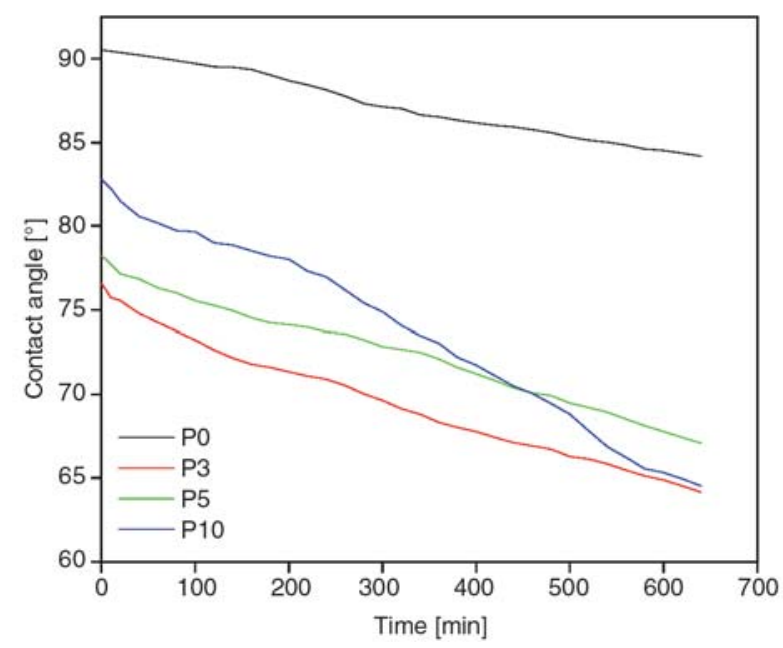

Figure 12. Variation contact angle of water with respect to time

decreased continuously with the contact time. In this context, the filler addition in PS may also bring about some surface restructuring. In addition to this the presence of carbon dioxide in the atmosphere may also affect the lowering of contact angles. Also the presence of filler particles on the surface may lower the contact angle over a period of time.

\section{Conclusions}

Polymer induced crystallization technique is employed to prepare nanoparticles of calcium phosphate with spherical morphology. The particles were incorporated in polystyrene by the melt blending technique. The transmission electron microscopic studies of the composites revealed good dispersion upto $5 \%$ of the filler loading. Contact angle measurements of polystyrene/calcium phosphate nanocomposites with water and methylene iodide were studied to know surface properties of the composites. The contact angles for water got decreased while the reverse trend was shown by methylene iodide. The hydrophilic nature of the composites increased with the addition of nanofillers due to presence of fillers on the surface. The solid surface free energy of the composites decreased and thereby increases the work of adhesion. The interaction between the liquid and solid surface became high compared to the neat polymer. With respect to time the contact angle of water decreased for sometime and leveled off which indicated some interaction between the surface and water. Methylene iodide showed less negative value for the spreading coefficient than water and it 
is a good wetting agent than water. Finally the particle dispersion in the matrix affected the various parameters analysed as each one of them changed according to the filler loading. Other physical and chemical properties will be undertaken to evaluate the viscoelastic and thermal behaviour of composites.

\section{References}

[1] Rozenberg B. A., Tenne R.: Polymer-assisted fabrication of nanoparticles and nanocomposites. Progress in Polymer Science, 33, 40-112 (2008).

[2] Wu Y-P., Zhang L-Q., Wang Y-Q., Liang Y., Yu D-S.: Structure of carboxylated acrylonitrile-butadiene rubber (CNBR): Clay nanocomposites by co-coagulating rubber latex and clay aqueous suspension. Journal of Applied Polymer Science, 82, 2842-2848 (2001).

[3] Krishnamoorti R., Vaia R. A., Giannelis E. P.: Structure and dynamics of polymer-layered silicate nanocomposites. Chemistry of Materials, 8, 1728-1734 (1996).

[4] Zanetti M., Lomakin S., Camino G.: Polymer layered silicate nanocomposites. Macromolecular Materials and Engineering, 279, 1-9 (2000).

[5] Vaia R. A., Giannellis E. P.: Lattice model of polymer melt intercalation in organically-modified layered silicates. Macromolecules, 30, 7990-7999 (1997).

[6] Liu L. M., Qi Z. N., Zhu X. G.: Studies on nylon 6/clay nanocomposites by melt-intercalation process. Journal of Applied Polymer Science, 71, 1133-1138 (1999).

[7] Kato M., Usuki A., Okada A.: Synthesis of polypropylene oligomer- clay intercalation compounds. Journal of Applied Polymer Science, 66, 1781-1785 (1997).

[8] Kwok D. Y., Lin R., Mui A. W., Neumann A. W.: Low-rate dynamic and static contact angles and the determination of solid surface tensions. Colloids and Surfaces, A: Physicochemical and Engineering Aspects, 116, 63-67 (1996).

[9] Kwok D. Y., Neumann A. W.: Contact angle interpretation in terms of solid surface tension. Colloids and Surfaces, A: Physicochemical and Engineering Aspects, 161, 31-38 (2000).

[10] Grundke K., Bogumil T., Gietzelt T., Jacobasch H-J., Kwok D. Y., Neumann A. W.: Wetting measurements on smooth, rough and porous solid surfaces. Progress in Colloid and Polymer Science, 101, 58-67 (1996).

[11] Kamusewitz H., Possart W.: Wetting and scanning force microscopy on rough polymer surfaces: Wenzel's roughness factor and the thermodynamic contact angle. Applied Physics, A: Materials Science and Processing, 76, 899-904 (2003).

[12] Vaia R., Ishii H., Giannelis E. P.: Synthesis and properties of two-dimensional nanostructures by direct intercalation of polymer melts in layered silicates. Chemistry of Materials, 5, 1694-1696 (1993).
[13] Manias E., Chen H., Krishnamoorti R., Genzer J., Kramer E. J., Giannelis E. P.: Intercalation kinetics of long polymers in $2 \mathrm{~nm}$ confinements. Macromolecules, 33, 7955-7966 (2000).

[14] Park S-J., Jin J-S.: Effect of silane coupling agent on interphase and performance of glass fibers/unsaturated polyester composites. Journal of Colloid and Interface Science, 242, 174-179 (2001).

[15] Saihi D., El-Achari A., Ghenaim A., Caze C.: Wettability of grafted poly(ethylene terephthalate) fibers. Polymer Testing, 21, 615-618 (2002).

[16] Yeh J-M., Chen C-L., Huang C-C., Chang F-C., Chen S-C., Su P-L., Kuo C-C., Hsu J-T., Chen B., Yu Y-H.: Effect of organoclay on the thermal stability, mechanical strength, and surface wettability of injectionmolded ABS-clay nanocomposite materials prepared by melt intercalation. Journal of Applied Polymer Science, 99, 1576-1582 (2006).

[17] Rogers K., Takacs E., Thompson M. R.: Contact angle measurement of select compatibilizers for polymersilicate layer nanocomposites. Polymer Testing, 24, 423-427 (2005).

[18] Rahimi A., Gharazi S., Ershad-Langroudi A., Ghasemi D.: Synthesis and characterization of hydrophilic nanocomposite coating on glass substrate. Journal of Applied Polymer Science, 102, 5322-5329 (2006).

[19] Chen X., You B., Zhou S., Wu L.: Surface and interface characterization of polyester-based polyurethane/ nano-silica composites. Surface and Interface Analysis, 35, 369-374 (2003).

[20] Robinette E. J., Palmese G. R.: Synthesis of polymerpolymer nanocomposites using radiation grafting techniques. Nuclear Instruments and Methods in Physics Research, Section B: Beam Interactions with Materials and Atoms, 236, 216-222 (2005).

[21] Wu T-M., Wu C-W.: Surface characterization and properties of plasma-modified cyclic olefin copolymer/layered silicate nanocomposites. Journal of Polymer Science, Part B: Polymer Physics, 43, 2745-2753 (2005).

[22] Park S. J.: Interfacial forces and fields: Theory and applications. Marcel Dekker, New York (1999).

[23] Israelachvili J.: Intermolecular and surface forces. Academic Press, London (1992).

[24] Young T.: An essay on the cohesion of fluids. Philosophical Transactions of the Royal Society of London, 95, 65-87 (1805).

[25] Fox H. W., Zisman W. A.: The spreading of liquids on low-energy surfaces. III. Hydrocarbon surfaces. Journal of Colloid Science, 7, 428-442 (1952).

[26] Fowkes F. M.: Additivity of intermolecular forces at interfaces. I. Determination of the contribution to surface and interfacial tensions of dispersion forces in various liquids. The Journal of Physical Chemistry, 67, 2538-2541 (1963).

[27] Owens D. K., Wendt R. C.: Estimation of the surface free energy of polymers. Journal of Applied Polymer Science, 13, 1741-1747 (1969). 
[28] Kaelble D. H.: Dispersion-polar surface tension properties of organic solids. The Journal of Adhesion, 2, 66-81 (1970).

[29] Wu S.: Polymer interface and adhesion. CRC Press, Boca Raton (1973).

[30] Ko Y. C., Ratner B. D., Hoffman A. S.: Characterization of hydrophilic-hydrophobic polymeric surfaces by contact angle measurements. Journal of Colloid and Interface Science, 82, 25-37 (1981).

[31] Lin J. W-P., Dudek L. P., Majumdar D.: Wetting properties of homopolymers and copolymers of pentafluorostyrene and methylacrylate and homopolymer blends. Journal of Applied Polymer Science, 33, 657667 (1987).

[32] El-shimi A., Goddard E. D.: Wettability of some low energy surfaces. I. Air/liquid/solid interface. Journal of Colloid and Interface Science, 48, 242-248 (1974).

[33] Pyter R. A., Zografi G., Mukherjee P.: Wetting of solids by surface-active agents: The effects of unequal adsorption to vapor-liquid and solid-liquid interfaces. Journal of Colloid and Interface Science, 89, 144-153 (1982).
[34] King R. N., Andrade J. D., Ma S. M., Gregnois D. E., Brostrom L. R.: Interfacial tensions at acrylic hydrogel-water interfaces. Journal of Colloid and Interface Science, 103, 62-75 (1985).

[35] Dupré A.: Theorie mechanique de la chaleur. Gauthier-Villars, Paris (1869).

[36] Girifalco L. A., Good R. J.: A theory for the estimation of surface and interfacial energies. I. Derivation and application to interfacial tension. Journal of Physical Chemistry, 61, 904-909 (1957).

[37] Jordan J., Jacob K. I., Tannenbaum R., Sharaf M. A., Jasiuk I.: Experimental trends in polymer nanocomposites-A review. Materials Science and Engineering, A, 393, 1-11 (2005).

[38] Lavielle L., Schultz J.: Surface properties of graft polyethylene in contact with water: I. Orientation phenomena. Journal of Colloid and Interface Science, 106, 438-445 (1985).

[39] Ruckenstein E., Lee S. H.: Estimation of the equilibrium surface free energy components of restructuring solid surfaces. Journal of Colloid and Interface Science, 120, 153-161 (1987). 